Rhode Island College

Digital Commons @ RIC

\title{
Assessing Nurses' Knowledge of Noise in the Intensive Care Unit: An Educational Intervention Project
}

Jennifer Fortes

Follow this and additional works at: https://digitalcommons.ric.edu/etd

Part of the Nursing Commons

\section{Recommended Citation}

Fortes, Jennifer, "Assessing Nurses' Knowledge of Noise in the Intensive Care Unit: An Educational Intervention Project" (2021). Master's Theses, Dissertations, Graduate Research and Major Papers Overview. 379.

https://digitalcommons.ric.edu/etd/379

This Major Paper is brought to you for free and open access by the Master's Theses, Dissertations, Graduate Research and Major Papers at Digital Commons @ RIC. It has been accepted for inclusion in Master's Theses, Dissertations, Graduate Research and Major Papers Overview by an authorized administrator of Digital Commons @ RIC. For more information, please contact digitalcommons@ric.edu. 
Assessing Nurses' Knowledge of Noise in the Intensive Care Unit: An Educational Intervention Project

by

Jennifer Fortes

A Major Paper Submitted in Partial Fulfillment

of the Requirements for the Degree of

Master of Science in Nursing

in

The School of Nursing

Rhode Island College

2021 


\begin{abstract}
Noise in the intensive care unit (ICU) has been studied for over thirty years, but it continues to be a significant problem and a top complaint among patients. Staff members are now reporting detrimental health effects from excessive noise. One of the significant factors of inadequate noise control in the ICU is that nurses have insufficient awareness regarding the hospital noise issue and its negative impact on health status. The level of knowledge of clinical staff on the topic of noise is not known. A quality improvement project to explore noise in the ICU could facilitate better understanding of the phenomenon and formulation of new ways to continue to reduce noise at a community hospital in Massachusetts. The purpose of this quality improvement project was to evaluate nurses' knowledge of the potentially harmful effects of noise on patients as well as on nurses, to identify opportunities for improvement of the environment, and to conduct an educational intervention aimed at reducing noise in the intensive care unit. The methodology for this project included a pre-test, followed by an educational session, and completion of a post-test. The participants included registered nurse staff members in the Intensive Care Unit (ICU) and the Critical Care Unit (CCU). Exclusion criteria included staff members who are not registered nurses. The project posed minimal risk. No identifying or biographical data was collected, and results included analysis of aggregate data. Descriptive statistics were used to assist with analysis. Results were disseminated to the staff of the ICU and CCU, posted on a bulletin board in the critical care area, presented as a poster presentation at the Spring RIC MSN Symposium, and available as a manuscript on the RIC Digital Commons.
\end{abstract}




\section{Acknowledgements}

I would like to thank Dr. Molloy and Dr. Costello for their time and dedication. It was a major factor in this project being as successful as it was. I would also like to thank my family. Their unwavering support on a daily basis was a major building block for this project's success. To my little guy Ethan, thank you for showing me unconditional love and helping me push forward every day. 


\section{Table of Contents}

Background/Statement of the Problem.........................................

Literature Review...........................................................

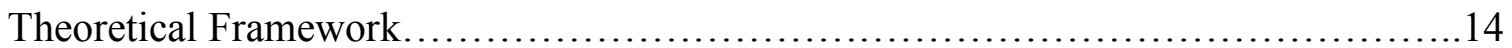

Method.................................................................... 17

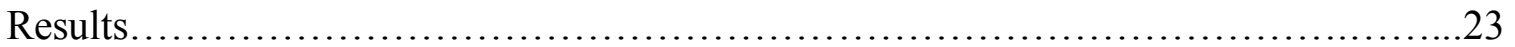

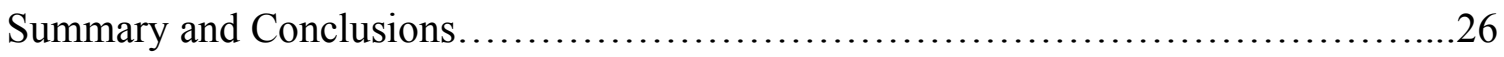

Recommendations and Implications for Advanced Nursing Practice..................30

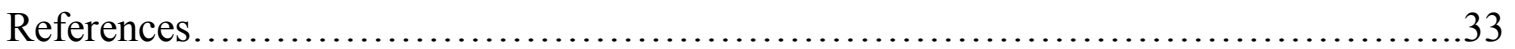

Appendices................................................................ 36 
Assessing Nurses' Knowledge of Noise in The Intensive Care Unit: An Educational Intervention Project

\section{Background/Statement of the Problem}

Noise in a busy acute care hospital is understandably present, especially in an Intensive Care Unit (ICU). This special care area of a hospital is designed to treat patients who are at substantial risk of death. Typical ICU's consist of multiple individual patient rooms containing equipment needed to treat these critically ill patients. These rooms are often bombarded with an array of noises including buzzers, pagers, telephones, and alarms. In addition, background sound often includes the conversation of medical professionals, patients, and families. These noises are often unpredictable and unexpected, as they have numerous sources. This can create a highly unpleasant and unhealthy environment. Florence Nightingale (1860) recognized noise as a health hazard stating, "Unnecessary noise, then, is the most cruel absence of care which can be inflicted either on sick or well."

A variety of types of noises exist in the hospital, including noise such as conversations, equipment alarms, telephones, pagers, televisions, closing doors, and staff activities (Konkani \& Oakley, 2012). These sounds are often undesired and disturbing, but can also be subjective based on the individual's personality, attitudes, and even cultural factors (Al-Tarawneh et al., 2020). Noise at high levels has been proven to have adverse effects on a patient's cardiovascular response, wound healing, gastric activity, and pain management. Other changes associated with noise in the intensive care units include sleep disturbances and increased risk of ICU psychosis (Hsu et al., 2012). All 
these factors can contribute to decreased healing for patients as well as increased risk of harm.

Research of effects of hospital noise on patients has been well studied over the last ten years, but an equally important aspect of this topic is the effect of noise on staff members. Excessive noise can lead staff to experience anxiety, increased stress, annoyance, and increased rates of burnout, thus possibly putting patients at risk for potential medical or nursing errors (Hsu et al., 2012). Research has shown that stress and job satisfaction can be influenced by environmental factors such as high noise levels, producing sensory overload (Ryherd et al., 2012).

Therapeutic procedures aimed at reducing the harmful effects of noise are continuously being developed. Noise in the ICU has been studied for over thirty years, yet we continue to struggle with this concept. Noise levels in the hospital have continued to be a top complaint among patients. Staff members are now reporting irritation, fatigue, and decreased ability to orally communicate when high levels of noise are present (Ryherd et al., 2012). Many strategies to reduce noise in the ICU have been developed over the last thirty years, including architectural adjustments, implementation of quiet time, and staff behavior analysis. These have demonstrated some success, but it has been noted that one of the significant factors of inadequate noise control in the ICU is that nurses have insufficient awareness regarding the hospital noise issue and its negative impact on health status (Al-Tarawneh et al., 2020). The degree to which clinical staff have knowledge on the topic of noise (Johansson et al., 2016) is unknown. This information could help the medical profession better understand and formulate new ways to continue to reduce noise (Al-Tarawneh et al., 2020). Therefore, the purpose of this 
quality improvement project will be to evaluate nurse's knowledge of noise, to assess the potentially harmful effects of noise on patients as well as nurses, to identify opportunities for improvement, and to conduct an educational program aimed at reducing noise in the intensive care unit. Next, a review of the literature will be provided. 


\section{Literature Review}

A review of the literature was conducted using CINAHL, PubMed, and Ovid. Searches for literature included the keywords hospital noise, hospital noise impacts, intensive care unit, nurses' knowledge, nurses' attitudes, and educational intervention. Limits to the literature reviewed were only those published in English and included only literature from the last 12 years, 2008-2020.

\section{Noise}

\section{Background}

Noise has been described as an undesirable sound, lacking harmony and rhythm. Similarly, noise pollution has been defined as a level of environmental noise that is disturbing and obtrusive (Khademi \& Imani, 2015). Noise does have a subjective component depending on its properties, and perception of noise may be influenced by individual sensitivities (Al-Tarawneh et al., 2020). It could be continuous, fluctuating, or intermittent, but ultimately all kinds of noise have been shown to have a negative impact on human health status whether the effects are immediate or long term (Konkani \& Oakley, 2012). These effects must be taken seriously, especially in caring for the most critically ill and vulnerable patients. Noise can be measured in many ways. A common unit of measurement is the decibel $(\mathrm{dB})$, which is a measurement of the energy contained in noise relative to the very minimum amount of energy average humans can detect (Hsu et al., 2012). Others include C-weighting (dBC), which is applied to very loud noises. Sound frequency is measured in Hertz $(\mathrm{Hz})$, and is related to human perception of pitch. As discussed, human perception of noise is highly complex. 


\section{Hospital Noise Clinical Impact}

Critical care units can often be hectic, chaotic, and generally over-stimulating, which can affect patient outcomes. The Joint Commission (TJC), an organization that certifies and accredits United States (US) healthcare organizations, has adopted reducing harm associated with clinical alarms as a National Patient Safety Goal (TJC, 2019). Doing so identifies noise as a potential risk factor for medical and nursing errors. To accomplish this patient safety goal, a few steps are recommended. This includes establishing alarm system safety as a hospital priority, identifying most important alarms to manage, establishing policies to determine which alarms will be most significant, and educating staff on strategies to reduce clinical alarms (TJC, 2019).

Increased noise levels in critical care units may lead to sleep deprivation, decreased healing, increased pain perception, increased delirium, and increased length of stay (Goeren et al., 2018). According to a study by Konkani and Oakley (2012), 34\% of noise in ICUs is avoidable, and $28 \%$ is partially avoidable. The noise may be attributed to operational factors such as staff conversation, equipment, cardiac monitors, and intravenous infusion pumps or to structural noise such as ventilation, air conditioning, and opening and closing of doors. One of the key roles of nurses is to manage the environment in the ICU to prevent negative outcomes and promote optimal health and healing. Therefore, knowledge of the important negative environmental stimuli and its effect on patients is critical and essential for all nurses involved. Al-Tarawneh et al. (2020) stated that a significant factor of inadequate noise control in the ICU was nurses' insufficient awareness regarding the hospital noise issue and its negative impact on health 
status. In a similar study, Johansson et al. (2016) found ICU nurses' knowledge of sound, noise, and their effects was deficient.

Many attempts to reduce noise in the ICU have focused on staff knowledge and behavior. These have included educational programs, implementation of guidelines, patient comfort packs including ear plugs and headphones, and sound detector controllers (Johansson et al., 2016). In a study by Conner and Ortiz (2009), it was demonstrated that after implementation of a quiet time intervention, overall noise decreased and Press Ganey patient satisfaction scores increased from the $2^{\text {nd }}$ percentile to the $95^{\text {th }}$ percentile. Also detailed in the study was that an important aspect of the results was education of the visitors, patients, and staff. Still, a study by Eliassen and Hopstock (2011), showed that only $22 \%$ of ICU nurses avoided bedside conversations at night and only about $10 \%$ of ICU nurses reduced alarm levels on monitors and ventilators during the day. Raising awareness and sensitivity of the staff is essential to counteracting noise pollution in the ICU.

\section{Sources of noise}

In the intensive care unit, complex biomedical equipment is used extensively and is essential to the monitoring of patients with complicated and serious medical conditions. These pieces of equipment are utilized to support patients' vital functions. Over time, developments in medical knowledge and technology in healthcare have resulted in increased noise levels in ICUs and other hospital areas. For example, alarms for cardiac monitors, mechanical ventilators, infusion pumps, and suction machines are used routinely. Also, staff activities have been identified as a significant contributor to increased noise levels in the ICU (Al-Tarawneh et al., 2020). Conversations between ICU 
staff, medical professionals, and visitors occur as well as caregiving activities such as hand washing, opening disposable equipment packages, storage drawers, closing doors, falling objects, telephones, pagers, and televisions (Goeren et al., 2018).

\section{Sound measurement}

Three expert organizations provide slightly variable standards for noise in acute care settings, recommending a range of no greater than 30-35 dB (decibels) during the night and no greater than $35-45 \mathrm{~dB}$ during the day. According to the World Health Organization (WHO), an international intergovernmental health organization, noise levels should not exceed $35 \mathrm{dBs}$ at night and $40 \mathrm{dBs}$ during the day (Darbyshire \& Young, 2013). The Environmental Protection Agency (EPA), a US governmental agency which regulates national environmental health, recommends the sound level limits of $35 \mathrm{dBs}$ at night, but allows increased sound level limit compared with the WHO to $45 \mathrm{dBs}$ at night. The International Noise Council (INC), a non-profit organization of professional Noise Control Engineers, recommends that environmental noise in the ICU not exceed $35 \mathrm{~dB}$ at night, $40 \mathrm{~dB}$ at sunset, and $45 \mathrm{~dB}$ during the day. An example of a sound source with a sound pressure level of $35 \mathrm{dBs}$ would be a very quiet room fan at low speed.

According to Konkani \& Oakley (2012), beginning at $40 \mathrm{dBs}$, learning or concentrating is possible, but distraction occurs. This is a key risk factor for patients because noise levels above $40 \mathrm{dBs}$ pose a higher potential for error by nurses due to barriers to concentration. Furthermore, at $45 \mathrm{~dB}$, noise of normal living occurs including talking, or radio in the background. People tend to raise their voice when background noise exceeds $45-50 \mathrm{~dB}$, thus increasing the overall noise level. A noise level of $55 \mathrm{dBs}$ is equivalent to a noisy vacuum cleaner, a noisy lawn mower is equivalent to $60 \mathrm{dBs}$, and 
$85 \mathrm{dBs}$ is equivalent to a chainsaw. In a study by Darbyshire et al. (2013), sound levels in ICUs in the United Kingdom were consistently found to be above WHO recommended limits and noise levels above $60 \mathrm{dBs}$ occurred every minute.

\section{Effects of noise}

Physiological effects. The human body responds to noise in the same way that it responds to stress (Choiniere, 2010). Over time, just as with chronic stress, excessive noise can impair health. A multitude of adverse physiological effects have been detailed in the literature. Some of these are cardiovascular disturbances including increased prevalence of hypertension and ischemic heart disease (Choiniere, 2010).

An established relationship between vasoconstriction and increased noise intensity was described in a review study by Hsu et al (2012) at noise levels greater than $70 \mathrm{dBs}$. The researchers found that exposure to noise levels of $90 \mathrm{dBs}$ could cause immediate vasoconstriction with a recovery time of twenty-five minutes. Hsu et al. (2012) also detailed the correlation between noisy periods in a Critical Care Unit (CCU) and the frequency of arrhythmias and the patients' state of anxiety. Twenty-five male patients were exposed to one-minute noise recordings equivalent to $55 \mathrm{dBs}$ or greater, and it was revealed that anxiety was heightened and the number of arrythmias increased. Overall, Hsu et al. (2012) found that patients exposed to loud noise in ICUs experienced common physiological changes such as increased heart rate, metabolism, and oxygen consumption.

Choiniere (2010) noted other physiological effects associated with increased hospital noise such as, sleep disturbances, hearing loss, and impaired wound healing. 
Additionally, the researcher found that some of the psychosocial effects of noise included increases in miscommunication and perceived annoyance.

Sleep disturbance. Sleep has two main functions, conservation and restoration of energy. It is necessary for physiologic restoration and maintenance of cognitive and emotional well-being. During sleep, noise can affect brain activity and cardiovascular function through elevated heart rate and blood pressure thus causing arousal (Fillary et al., 2015). In a study by Darbyshire et al. (2013), the authors stated that over thirty percent of patients treated in ICU's become confused or experience delirium. These patients have longer hospital stays and higher morbidity and mortality. Risk factors for the development of ICU related delirium are sedation use and invasive procedures, but environmental factors, including noise-induced sleep disturbance, were also linked to delirium (Darbyshire et al., 2013).

Noise and Staff Response. An interesting and important area involved in the topic of noise is its effects on staff members. Research focus on the topic of noise has mostly discussed the effects of noise on patients, not necessarily including its effects on staff members. As discussed previously, noise has adverse physiological effects. This applies to patients as well as staff members.

Interestingly, in addition to physiological effects of noise such as elevated heart rate, psychological effects occur as well. These can include headaches, anxiety, emotional stress, and burnout (Konkani et al., 2014). In a review study by Ryherd et al. (2012), stress, satisfaction, and psychosocial conditions were found to be influenced by sensory overload due to environmental factors such as high noise levels. Hospitals are understandably stressful work environments, even absent from noise. Stressors are caused 
by a multitude of demands, such as what we have a need for and what we are capable of achieving as well as what our environment provides and what it demands of us (Ryherd et al., 2012). Subsequently, occupational stress is derived from workplace conditions, which can include noise, shift times, amount of work hours, level of responsibility, and place of work (Ryherd et al., 2012). Ryherd et al. (2008) surveyed 47 nurses in a neurologic intensive care unit and found that $91 \%$ believed that noise negatively affected them in their daily work environment. Many reported experiencing noise-induced stress symptoms such as irritation, fatigue, and headaches. The noise in this particular unit averaged between 53 and $58 \mathrm{dBs}$ (Ryherd et al., 2008).

In a review study by Choinere (2010), it was reported that nurses spend far more time in the ICU than patients, meaning the nursing staff experience more cumulative exposure to excessive noise levels than patients, yet the long-term effects of noise on nursing staff is not well understood. Non-hospital literature contains evidence suggesting that environmental sensory overload can disrupt processing of stimuli and information. Ryherd et al. (2012) described the overload hypothesis, which suggests that humans have a finite capacity for processing stimuli and cope with overload by utilizing selective attention and ignoring low-priority inputs. Over time, the authors postulate that repeated exposure of staff to sensory overload can lead to medication errors, misjudgment, or even compassion fatigue. They hypothesize that this in turn compromises patient safety.

\section{Strategies for noise elimination}

A multitude of strategies to reduce noise in the ICU have been discussed in the literature. These strategies can be categorized into three types of interventions: architectural-acoustic design, equipment design, and staff education. The use of 
architectural-acoustic design includes interventions such as sound absorbent material for ceilings, walls, and floors (Milette, 2010). While these interventions have been reported effective in noise reduction strategies, they can be difficult to implement architecturally, and can be very costly (Milette, 2010). The use of quiet equipment is another potential solution, but this equipment is often more costly than a more conventional type. Hospitals are required to be financially conservative in every aspect making purchase of quieter equipment unlikely. Another important point in noise reduction efforts is the focus of monitor alarm reduction measures.

In a study by Sendelbach et al. (2015), a quality improvement project was conducted on a cardiovascular care unit to decrease electrocardiographic nuisance alarms. Staff education, as well as a bundled approach, was used that included eliminating duplicate alarms, customizing alarms, changing electrocardiography electrodes daily, skin preparation, and using disposable electrocardiography leads. The results of the study showed an $88.5 \%$ reduction in mean electrocardiographic alarm signals per day.

Goeren et al. (2018) utilized another noise reduction strategy by implementing an improvement plan including a quiet time initiative. Staff, patient, and family education was completed and quiet time was initiated one week later. Decibel data was collected for sixty days. Results of this study revealed statistically significant reduction in noise levels compared to baseline data. The authors recognized that the primary focus of this study was educating the staff about noise levels in the intensive care environment. They utilized a journal club to introduce peer-reviewed articles to staff and also had a discussion to incorporate this evidence for best practice. Researchers of the study described that small changes in practice by staff led to peak noise reduction during the allotted quiet time. 
Other equally important noise reduction strategies include the use of noise canceling headphones and earplugs to improve the subjective perception of noise by patients (Milette, 2010). Given that staff have been recognized in the literature as being significant noise creators, the most obvious way to decrease noise would be to increase staff awareness. Doing so, along with education about the effects of noise, would hopefully lead to daily practice change, thus reducing noise levels. In a study by Milette (2010), a quality improvement project was implemented including a noise awareness educational program. Baseline noise levels were measured in a neonatal intensive care unit (NICU), and pre-intervention mean noise levels were found to be significantly higher than recommended. Post intervention measurements did not appear to be statistically significant which ultimately was attributed to a significant increase in activity level. After accounting for increase in activity level, it was found that noise levels did significantly decrease post intervention, revealing the important effects of raising staff awareness on noise reduction.

In an article by Vijay (2014), the topic of reducing and optimizing hospital noise is discussed. The article outlines that a hospital must strive to create a quiet and calm environment to promote healing and recovery while patients and families are dealing with stess from illness, medical visits, hospitalizations, and bereavement (Vijay, 2014). The impact of hospital noise was also described, andthe startle reflex as a potential physiologic effect of noise was discussed. The startle reflex includes reactions such as facial grimacing, increasing blood pressure, higher respiratory rate, increased heart rate, and vasoconstriction (Vijay, 2014). This is interesting because very often when humans hear a loud noise, they may jump, and say "Oh that scared me!" This reaction is very true 
and a realistic possibility for our patients as well as staff. With this repeated type of exposure to noise or activation of the "fight or flight" response in our bodies serious physical consequences can occur, including chronic stress response and increased cortisol. Strategies to eliminate excessive noise listed in the article consisted of evaluating current patient safety standards, analyzing current hospital noise through patient satisfaction surveys, as well as measuring decibel levels, converting a centralized nursing station to a decentralized nursing station, and providing guidance and instruction to staff members on importance of maintaining appropriate noise levels (Vijay, 2014).

\section{Discussion}

In summary, noise present in the hospital environment has a multitude of adverse physiological effects on patients as well as staff members. Such effects can produce long term chronic health changes, which is an alarming and understudied topic. An intervention that has proven effective and is relatively low in cost is increasing amounts of staff education on the topic of noise pollution. Doing so could raise awareness of effects on patients as well as staff and potentially lead to daily practice change, decreasing levels of noise in the hospital environment. An understanding of the baseline of staff's current knowledge is needed to understand and confirm the need for intervention. The purpose of this proposed quality improvement project is to evaluate nurse's knowledge of noise, the potentially perceived harmful effects of noise on patients as well as nurses, to identify opportunities for improvement, and to conduct an educational program aimed at reducing noise in the intensive care unit. 


\section{Theoretical Framework}

Quality improvement projects often pose unique challenges in terms of development. They require multi-faceted interventions that extend over a period of time and typically involve a team with few resources for data collection. Crucial to project success is how each phase of the project and intervention build upon each other. The Logic Model developed by the W. K. Kellogg Foundation was used as the theoretical framework for development of this educational intervention. The logic model guides the

development of a project by describing how it will work, overall outcome goals, and what resources will be needed (W. K. Kellogg Foundation, 2004). This model is ultimately comprised of a combination of a few factors. It begins with what inputs or resources needed for the project, including activities being utilized, then progresses to how you will achieve the intended results, and outcomes being desired. The logic model guides project teams in planning, implementing, and evaluating projects that will achieve the desired results.

The initial phase of the logic model is the planning work phase. This phase requires evaluation of resources and any barriers to those resources, which could enable or limit the program itself. This phase is also referred to as the input of the project (W. K. Kellogg Foundation, 2004). Some of this project's resources will include registered nurse staff participants in the ICU as well the actual hospital environment where the project will take place. Also, other project resources will include the amount of time needed for intervention, and the nurse managers' as well as administrators' approval of the project. All resources will be detailed and sought out prior to the implementation of the project. 
The next step in the Logic Model is also part of the planning work phase, but is referred to as activities, which is using the resources previously discussed, and creating the program activity. In this phase, the project team determines what the educational information would be and how it would be delivered. This step will be the actual project development and includes using the tools and resources to complete this. For this project, the activity will be a ten to fifteen-minute educational intervention for critical care nurses. Knowledge change will be evaluated through a pre-and post-test questionnaire. Using these steps of the logic model will help to create a seamless transition among phases for successful planning of this project. This will lead to overall successful implementation of the project, with achievable outcomes, and quality improvement within the organization.

The next phases of this framework are related to the intended results and outcomes of the project. The first step in this phase will be related to actual outputs, which means delivering the intended information to create an outcome. For this project, the outputs are being used by providing the intended information for critical care nurses to receive and understand as well as to complete the questionnaires. Also, a factor in outputs of the Logic model is the quality of the program as well as number of participants reached. This will mean completing the educational project in an efficient, but desirable amount of time with an adequate number of nurses, and assessment of knowledge improvement by analysis of the questionnaires. The second step of this phase involves the actual outcomes to be achieved. In this project, the outcomes desired will be related to improved knowledge of noise pollution as well as interventions for noise reduction in the critical care setting. This knowledge change will be examined as aggregate data through a post intervention test. The final step of this phase is the impact of this individual project 
being developed. This will be related to the overall goals of the project. For this project, it is anticipated that by assessing nurses' knowledge on noise, and delivering education on the topic, this will aid in filling knowledge gaps, which will potentially lead to daily practice change and reduction in noise levels. This framework will help guide each phase of the educational project being proposed, starting with gaining resources, progressing to implementation of the program, and finally the evaluation of overall knowledge. Next, the methodology for this project will be presented. 


\section{Purpose}

\section{Method}

The purpose of this quality improvement project was to evaluate nurses' knowledge of the potentially harmful effects of noise on patients as well as on nurses, to identify opportunities for improvement in noise levels in the environment, and to conduct an educational intervention aimed at reducing noise in the intensive care unit.

\section{Design}

The design of this quality improvement project was a pre-test/post-test educational intervention. The tests were used to evaluate the nurses' baseline knowledge on noise as well as knowledge improvement related to the educational intervention provided.

\section{Sample/Site}

The study used a non-probability, convenience sampling with the goal of including all staff nurses working in the sixteen-bed critical care area at a three hundred twenty-eight bed community hospital in Massachusetts. Inclusion criteria included all registered nurses in the ICU and CCU. Exclusion criteria included non-registered nurse staff members in the same sixteen bed critical care area. It was anticipated that approximately thirty registered nurses met inclusion criteria.

\section{Measurement}

The evaluation of the baseline level of nurses' knowledge as well as knowledge improvement on the topic of noise in the ICU and CCU was assessed using a questionnaire created by this researcher. Guidelines for creation of this questionnaire as well as the educational intervention were obtained from a data driven methodology known as Six Sigma. This methodology is described as making business processes more 
effective and efficient by offering tools and techniques that reduce variance and eliminate defects, thus allowing organizations to create better products and services for their customers (Sixsigmadaily.com, 2021). Questions 1-2 in the questionnaire addressed the definition of noise. Questions 3-8 addressed the hospital clinical impact of noise, and questions 9-10 addressed strategies for improvement in noise.

These questions were developed based on the information that was presented in the educational session. A copy of the questionnaire is provided in Appendix D. Analysis of the pre-test questionnaire provided data on level of baseline knowledge of noise in the ICU. Analysis of the post-test questionnaire provided data measuring knowledge change post educational session. Descriptive statistics such as central tendencies of mean, median, and mode were used to assist with analyses. The questionnaire was piloted on four nurses with ICU experience prior to implementation of the quality improvement project which ensured reliability and validity of the survey questions.

\section{Institutional Review Board}

This project involved human subjects and was submitted for approval to the Rhode Island College (RIC) and Southcoast Institutional Review Boards (IRB). Minimal risk was present with this quality improvement project, and IRB designation of exemption was received from both Southcoast and RIC for this quality improvement project.

\section{Ethical/Human Subject Considerations}

Ethical considerations for this project were determined to be minimal. Administrative approval was obtained. The nurse manager who supervised the two critical care units, ICU and CCU, granted permission to complete the project and assured 
access to their nursing staff as well as assistance to allow staff the time to participate. Permission from the chief nursing officer (CNO) as well as the clinical trial coordinator was also obtained. The critical care nurse educator agreed to be the preceptor for this project. The documentation of the agreement is provided in Appendix E.

Minimal risk existed to the participants. No identifying biographical data was

collected. Pre and post tests were analyzed using aggregate data only. Descriptive statistics such as central tendencies of mean, median, and mode were used to assist with analysis. Confidentiality was maintained by keeping participant results anonymous with no identifying data as well as providing privacy while participants were completing the pre and posttest questionnaires. Research materials were stored in the nurse manager's office in a locked box with access only by the researcher and nurse manager. The data will be stored for three years in a locked secure environment following the completion of the study and will then be disposed of by shredding.

\section{Procedures}

The educational intervention was offered to all registered nurses in the critical care area including the Intensive Care Unit (ICU) and Coronary Care Unit (CCU) to ensure equal access. The identical educational sessions were repeated at different times throughout the day to accommodate interested nurses on off-shifts and encourage participation. The sessions were at 0900 , followed by an 1830 , and finally a 1930 session. Nurses were not allowed time off from work to attend the educational session, but the session was ten minutes in length and was conducted in a quiet corner of the unit, which provided both privacy and limitation of distraction, while also allowing nurses working that day the ability to participate. The educational sessions were also timed so that nurses 
could arrive for their shift ten minutes early and participate as well. This required utilization of their own time since they were not paid for time not scheduled.

An informational email (Appendix A) was sent to all ICU and CCU nurses on the unit which briefly described the project's purpose and proposed timeline. Then, a second informational letter (Appendix B) was sent via email to all nurses in the ICU and CCU. This email provided a more detailed overview of the procedures and use of the test results and was sent one month in advance of the program to allow the information to reach as many nurses as possible. It was explained in the email that participation in the research was voluntary, and no identifiable biographical data was collected. Informed Consent was implied when the nurse participant read the informational letter, completed the pretest, and attended the educational session which was stated in the informational letter. Another promotional strategy that was utilized was flyers which were posted in each unit two weeks in advance of the program and contained the information provided in the informational letter.

Implementation of the project began in February 2021 following IRB designation of exemption, beginning with the informational letter. After two weeks of dissemination of information about the project to potential participants, the dates and times for the educational sessions were established. Each proposed session began with a ten-question pre-test which was delivered the same day as the educational intervention. Each participant was provided the pretest and a clean pen. They completed it maintaining social distancing and standard COVID-19 precautions. The tests were anonymous and no participant identifiers were utilized. Participants then completed the same test post 
intervention also on the same day as the educational intervention. The pre and post tests were analyzed and aggregate data was utilized.

\section{Educational Session}

The educational program was developed and delivered to the nurses in the ICU and CCU. Multiple sessions were offered throughout the day to accommodate different staff shifts and to ensure participation from as many nurses as possible. The objectives for the educational program were developed based on the needs assessed in a thorough literature review. Informational content on the scope of the problem, impacts of noise pollution on patient outcomes and on the health of nurses, and strategies for noise reduction was obtained through the literature review. The researcher delivered the educational material at each session using a handout with opportunities for participant questions and discussion. A content outline of the proposed educational program is

provided in Appendix C. The content areas include background information on the scope and significance of noise, clinical impact of noise pollution on patients and staff in the acute care setting, and strategies to reduce ICU noise.

The educational session was conducted at the nurses' station on the ICU or CCU. Group sizes were somewhat flexible, but a maximum of six participants at each session were allowed to ensure adequate participant focus and COVID-19 spacing requirements. Social distancing guidelines were maintained. This included participants spaced six feet apart during the sessions, each person wearing a mask, being provided cleaned individual pens, and hand hygiene at the beginning and end of the sessions. The participants then received a post-intervention test to evaluate satisfactory knowledge improvement. Multiple sessions were offered throughout the day to accommodate different staff shifts 
and to ensure participation from as many nurses as possible. At the completion of the sessions, completed pre-and-post-tests were compared through data analysis.

\section{Data Analysis}

Descriptive statistics such as central tendencies of mean, median, and mode were used to assist with analysis to measure the effectiveness of the educational program. Outcome variables being used to determine effectiveness of the program were nurse participants' baseline knowledge of noise in the ICU and knowledge improvement post educational intervention. All questions were used to compare differences between nurse participants' knowledge of noise pre and post educational intervention. Pretest responses were compared and analyzed to post-education posttest responses utilizing percentages, percentiles, and total scores. Data is presented in the Results section.

\section{Plan for evaluation and dissemination}

This educational intervention was evaluated by examining how nurse participants' knowledge of noise has improved from the pre to post survey. Data analysis was completed using basic descriptive statistics and will be graphically displayed. Results were disseminated to the staff at the morning team huddles in each unit, as well as to the acute care hospital's professional development team which presently guides the education for nurses. Results were also posted on a bulletin board in the critical care area in an area accessible to all staff. A poster presentation of findings from this proposed project was presented to the Rhode Island College (RIC) faculty, students, and community at an Master's Project Symposium. The written Master's Project manuscript was posted on RIC's Digital Commons. 


\section{Results}

A total of sixteen $(\mathrm{N}=16,53.3 \%)$ nurses participated in the project of the thirty possible. All sixteen participants completed the pre-test, followed by the educational session, and the post test. This was all completed in the same day, at the time of the session in which they were participating. Analysis of the data has been completed utilizing measures of central tendency including mean score. All questions were presented as multiplechoice items. The details of the participants' answers on the pre and post tests can be found in Appendix F. Each multiplechoice question had one correct response. The questions were scored based on the number of correct responses. Table 1 reveals the percent of nurses participating in the study who answered each question with the appropriate response for both the pre and post tests. 


\section{Table 1:}

Pre/Post Test Appropriate Response Score Comparison

\begin{tabular}{|c|c|c|c|}
\hline Question & Pre-test (N=16) & Post-test (N=16) & Percent Increase \\
\hline $\mathbf{1}$ & $68.75 \%(11 / 16)$ & $100 \%(16 / 16)$ & $31.25 \%$ \\
\hline $\mathbf{2}$ & $93.75 \%(15 / 16)$ & $100 \%(16 / 16)$ & $6.25 \%$ \\
\hline $\mathbf{3}$ & $12.5 \%(2 / 16)$ & $87.5 \%(14 / 16)$ & $75 \%$ \\
\hline $\mathbf{4}$ & $25 \%(4 / 16)$ & $93.75 \%(15 / 16)$ & $68.75 \%$ \\
\hline $\mathbf{5}$ & $37.5 \%(6 / 16)$ & $81.25 \%(13 / 16)$ & $43.75 \%$ \\
\hline $\mathbf{6}$ & $25 \%(4 / 16)$ & $87.5 \%(14 / 16)$ & $62.5 \%$ \\
\hline $\mathbf{7}$ & $68.75 \%(11 / 16)$ & $93.75 \%(15 / 16)$ & $25 \%$ \\
\hline $\mathbf{8}$ & $75 \%(12 / 16)$ & $100 \%(16 / 16)$ & $25 \%$ \\
\hline $\mathbf{9}$ & $75 \%(12 / 16)$ & $100 \%(16 / 16)$ & $25 \%$ \\
\hline $\mathbf{1 0}$ & $68.75 \%(11 / 16)$ & $100 \%(16 / 16)$ & $31.25 \%$ \\
\hline
\end{tabular}

Ultimately, the scores were reviewed for total improvement in responses. In review of pre and post test scores, all demonstrated an improvement. The mean score for pre-test percentage of correct responses was 55\%. The mean score for post-test percentage of correct responses was $84.375 \%$. This represents a $29.375 \%$ increase post educational intervention. All responses for pre-tests can be found in appendix F, and all responses for post-tests can be found in Appendix G. These are listed in a table in a randomized format, as the pre and post tests were not matched for each participant to assure confidentiality. 
Upon further review of the data, Question 3 showed the greatest improvement in percentage of correct responses. This question was about World Health Organization (WHO) Guidelines for acceptable ranges of noise levels in the hospital. In pre-test responses, $12.5 \%$ or $2 / 16$ nurses responded with the appropriate response. In post-test responses, $87.5 \%$ or $14 / 16$ nurses responded with the appropriate response. This revealed an increase of $75 \%$.

Overall, question 5 received the lowest percentage of appropriate responses post intervention at $81.25 \%$, increased from $37.5 \%$ pre-test. This question was asking at what noise level does distraction start to occur while learning and/or concentrating. Question number 4, asking what noise level is that of normal living, also showed an increase in correct responses. In pre-test responses, four out of sixteen nurses (25\%) answered the appropriate response. In post-test responses fifteen out of sixteen nurses $(93.75 \%)$ answered the appropriate response. This revealed an increase of $68.75 \%$. Question 6, asking about average noise level at the nurses' station in the ICU, revealed a $62.5 \%$ improvement in post-test scores. In pre-test responses, four out of sixteen (25\%) participants, responded with the appropriate response. In post-test responses fourteen out of sixteen $(87.5 \%)$ responded with the appropriate response.

Next the summary and conclusions will be presented. 


\section{Summary and Conclusions}

A hospital intensive care unit (ICU) is designed to treat critically ill patients who need special care and treatment. Over time as medical advances have occurred, the complexity of the ICUs have increased as well. This includes use of numerous sophisticated pieces of medical equipment to support an array of physicians, nurses, technicians, and other staff. The increase in equipment and treatment modalities has dramatically increased noise in the ICU (Konkani \& Oakley, 2012), and its effects on patients and staff has become an important issue. Excessive noise is not only disturbing, but it can potentially interfere with proper patient care and safety. Research has identified that staff education can be highly effective in preventing noise in the ICU (Khademi \& Imani, 2015). In light of this relevant clinical need, a quality improvement project was developed by this investigator to evaluate nurses' knowledge of the potentially harmful effects of noise on patients as well as on nurses, to identify opportunities for improvement in noise levels in the environment, and to conduct an educational intervention aimed at reducing noise in the intensive care unit.

This educational program was conducted at a small community hospital in New England, and was evaluated using a pre/post-test model. The pre-tests were completed the same day as the intervention, prior to the educational session. The educational session was then completed in 10 minutes, and subsequently the participants completed the posttest. The pre and posttest answers were compared to assess for knowledge improvement post educational intervention.

Of the projected goal of thirty participants, a total of sixteen $(\mathrm{N}=16,53.3 \%)$ participated in the project. All sixteen participants completed the pre-test, followed by 
educational intervention, and then post-test. All components of the project were completed on the same day for each of the participants. All ten questions were multiple choice with one corresponding correct response. This was performed to assess for knowledge improvement post educational intervention. A review of pre and post test scores demonstrated an improvement for all participants. The mean score for pre-test percentage of correct responses was $55 \%$. The mean score for post-test percentage of correct responses was $84.375 \%$. This represents a $29.375 \%$ increase post educational intervention.

Questions 1 and 2 were questions that had relatively high pre-test appropriate responses, $68.75 \%$ and $93.75 \%$ respectively. These questions asked definitions of noise and units of measurement. These relatively high scores preeducational intervention reveal that a basic understanding of noise and what it is composed of is in fact well understood by nurses.

A question that revealed the highest percentage of knowledge improvement was question 3. This question asked about the World Health Organizations (WHO) recommendations for acceptable range of noise level in the hospital. In pre-test responses, $12.5 \%$ or $2 / 16$ nurses responded with the appropriate response. In post-test responses, $87.5 \%$ or $14 / 16$ nurses responded with the appropriate response. This revealed an increase of $75 \%$. These responses are interesting because while noise has been an important topic for many years, it is evident from this question that knowledge of noise regulations is not well known by nurses.

Question 5, which asks about what noise level distraction starts to occur while learning and/or concentrating, received the lowest percentage of appropriate responses 
post intervention at $81.25 \%$, increased from $37.5 \%$ pre-test. These responses provide interesting information because knowledge of when distraction begins to occur is important for daily practice. While optimizing patient care and safety, distraction is something that should not be afforded. While this question received the lowest score post-intervention, it may mean that it is something that will need to be reinforced over time with nurses.

Questions 8-10 were about strategies for noise reduction. These questions overall received high scores pre-intervention as well as post-intervention. This demonstrates that teaching on noise reduction strategies has been implemented thus far, and further education can continue to build on that knowledge foundation to further reduce instance of high noise levels.

In review of all ten questions, it is evident that basic knowledge of noise such as definition is understood by nurses, as well as noise reduction strategies. The biggest areas for improvement are in determining what actually constitutes as noise that is too loud, and how does that compare to regulations put in place by governing bodies such as WHO. Question 6, which asked what the average level of noise is near the nurses' station, four out of sixteen (25\%), responded with the appropriate response. This demonstrates that knowledge of how high the noise levels at the nurse's station is not actually well known, and further education is needed. After the educational intervention it revealed a $62.5 \%$ improvement in scores.

Some limitations were noted for this project. Project participation was lower than expected with a total of sixteen $(\mathrm{N}=16,53.3 \%)$ of the projected thirty participants. An informational email was provided a few weeks in advance as well as manager 
encouragement. Low participation may have been related to a lack of nurses checking their email regularly, and also possibly a lack of flyers on display in the units. Education was also provided during staff work times, which makes it difficult to participate. Also important in considering is the personal and professional stress being experienced because of the COVID-19 Pandemic. This may have made it more difficult for nurses to dedicate time for this project.

Overall, the educational project demonstrated success for nurses who participated. Improved knowledge regarding noise in the ICU was evident.

Next, recommendations and implications for advanced nursing practice will be presented. 


\section{Recommendations and Implications for Advanced Nursing Practice}

The Advanced Practice Registered Nurse (APRN) has a multitude of opportunities to identify gaps in education, as well as improvement of nursing practice. APRNs are leaders in demonstrating best practice in many areas of the healthcare system. They do this not only by utilizing evidencebased nursing in the daily care of their patients, but also for education of nursing staff for interventions. Educational interventions have been identified as an important, effective, and cost-effective way to provide instruction as well as promote practice change. The literature supports that noise in the ICU is an important topic that needs to remain a priority of the ICU staff in order to improve patient safety as well as satisfaction, but also to reduce incidence of burnout among ICU staff members. Enhancing nursing knowledge regarding noise the in ICU would eventually aid in creating a healing, pleasant, and more comfortable environment by eliminating the negative effects of this environmental stressor.

The APRN can assist in developing and implementing an educational program. This educational program in particular provides evidence based research regarding noise in the ICU, but also assists in implementing strategies for noise reduction. The APRN can utilize research to support staff behavior improvement strategies to reduce ICU noise levels. A study by Milette, 2010, revealed that a noise awareness educational program reduced mean noise levels in their neonatal intensive care unit (NICU). APRNs are pivotal at providing support for nursing practice change. THe APRN can educate the nurses on how to remain mindful of levels of noise in the ICU and provide reminders for interventions such as reduction in staff conversations at the centralized nurses' station. The APRN can also provide evidence to hospital value analysis committees for potential 
need for quieter equipment or increased signage in the hospital to promote a quiet environment.

This project helped to identify that there is a gap in knowledge regarding noise in the ICU. Further education regarding how much noise is too much noise should be provided. This project revealed that nurses have a baseline level of knowledge regarding noise, but knowledge was lacking regarding what actually constitutes as excessive noise and also at what level noise can negatively impact nurses in the workplace. The APRN can assist the nurse in identifying high levels of noise and provide education to promote noise reduction strategies.

The APRN can involve an interdisciplinary care team including physicians, nursing staff, hospital employees to further target measures aimed at reducing noise in the ICU. This topic has important implications for future nursing education including a focus on prevention of noise. Also important is this topic's implications for policy change in a health care system. This could include changes such evaluation of the hospital communication and paging system with the possibility of implementing policies to minimize overhead pages, especially at night.

This topic also has widespread population health implications. Increased rates of nurse burnout as well as stress and anxiety can lead to a nurse shortage, especially during an already stressful time such as the COVID-19 pandemic. Further focus on measures to reduce stress and anxiety related to noise in the ICU is an important research point.

Overall, future goals of research on this topic should include optimizing patient safety, assuring health equity, implementing evidence based research, and improving the workplace environment for nurses. Patient safety is of extreme importance and a 
benchmark looked at globally. Therefore, the ability to ensure it by implementing proven strategies for reducing noise is critical. Improving the environment for nursing practice is also an important benchmark which would prevent nurse burnout and stress and improve patient outcomes . 


\section{References}

Al-Tarawneh, O. M., Demeh, W. M., \& Yacoub, M. I. (2020). An assessment of nurses knowledge regarding noise in intensive care units in Jordan. International Journal of Africa Nursing Sciences, 12, 100183. doi: 10.1016/j.ijans.2019.100183

Choiniere, D. B. (2010). The Effects of Hospital Noise. Nursing Administration Quarterly, 34(4), 327-333. doi: 10.1097/naq.0b013e3181f563db

Connor, A. (2009). Staff Solutions for Noise Reduction in the Workplace. The Permanente Journal, 13(4). doi: 10.7812/tpp/09-057

Darbyshire, J. L., \& Young, J. D. (2013). An investigation of sound levels on intensive care units with reference to the WHO guidelines. Critical Care, 17(5). doi: $10.1186 / \operatorname{cc} 12870$

Eliassen, K. M., \& Hopstock, L. A. (2011). Sleep promotion in the intensive care unit-A survey of nurses' interventions. Intensive and Critical Care Nursing, 27(3), 138142. doi: 10.1016/j.iccn.2011.03.001

Fillary, J., Chaplin, H., Jones, G., Thompson, A., Holme, A., \& Wilson, P. (2015). Noise at night in hospital general wards: a mapping of the literature. British Journal of Nursing, 24(10), 536-540. doi: 10.12968/bjon.2015.24.10.536

Goeren, D., John, S., Meskill, K., Iacono, L., Wahl, S., \& Scanlon, K. (2018). Quiet Time: A Noise Reduction Initiative in a Neurosurgical Intensive Care Unit. Critical Care Nurse, 38(4), 38-44. doi: 10.4037/ccn2018219

Hsu, T., Ryherd, E., Persson Waye, K., \& Ackerman, J. (2012). Noise pollution in hospitals: Impact on patients. Journal of clinical outcomes management, 19(7), 301-309. 
Johansson, L., Knutsson, S., Bergbom, I., \& Lindahl, B. (2016). Noise in the ICU patient room - Staff knowledge and clinical improvements. Intensive and Critical Care Nursing, 35, 1-9. doi: 10.1016/j.iccn.2016.02.005

Khademi, G., \& Imani, B. (2015). Noise pollution in intensive care units: a systematic review article. Reviews in Clinical Medicine, 2(2), 58-64.

Konkani, A., Oakley, B., \& Penprase, B. (2014). Reducing Hospital ICU Noise: A Behavior-Based Approach. Journal of Healthcare Engineering, 5(2), 229-246. doi: $10.1260 / 2040-2295.5 .2 .229$

Milette, I. (2010). Decreasing Noise Level in Our NICU. Advances in Neonatal Care, 10(6), 343-351. doi: 10.1097/anc.0b013e3181fc8108

Nightingale, F. (1860). Notes on Nursing: What it is, and what it is not. A Celebration of Women Writers. http://digital.library.upenn.edu/women/nightingale/nursing/nursing.html\#IV

Ryherd, E. E., Okcu, S., Ackerman, J., Zimring, C., \& Persson Waye, K. (2012). Noise pollution in hospitals: Impacts on staff. Jcom Journal, 19(11), 491-500.

Ryherd, E. E., Waye, K. P., \& Ljungkvist, L. (2008). Characterizing noise and perceived work environment in a neurological intensive care unit. The Journal of the Acoustical Society of America, 123(2), 747-756. doi: 10.1121/1.2822661

Sendelbach, S., Wahl, S., Anthony, A., \& Shotts, P. (2015). Stop the Noise: A Quality Improvement Project to Decrease Electrocardiographic Nuisance Alarms. Critical Care Nurse, 35(4), 15-22. doi: 10.4037/ccn2015858

Sixsigmadaily.com. (n.d.). Retrieved February 16, 2021, from https:/www.sixsigmadaily.com/what-is-six-sigma/ 
The Joint Commission. (2019). 2019 National Patient Safety Goals. Retrieved April

1,2020, from https:/www.jointcommission.org/-

/media/tjc/documents/standards/national-patient-safety-

goals/historical/2019_hap_npsgs_final2.pdf?db=web\&hash=60F2AF29475AB23

50D631011595EBB3E

Vijay, S. (2014, September 10). Reduce and Optimize Hospital Noise with Six Sigma

Tools. Retrieved October 23, 2020, from

https://www.isixsigma.com/industries/healthcare/reduce-and-optimize-hospital-

noise-six-sigma-tools/

W.K. Kellogg. (2004, January). Logic model development guide. Retrieved from W.K.

Kellogg Foundation: www.wkkf.org 


\title{
Appendix A \\ Informational Email
}

\section{Hello Critical Care Nurses,}

My name is Jennifer Fortes; I am a Rhode Island College Nurse Practitioner student. I am in a master's level program, and I will be completing a quality improvement project on your floor. Attached to this email is an informational letter about the program. This letter will be available in the breakroom as well as in the manager's office. Time will be provided for completion of the program in Fall 2020. Participation in this program is voluntary.

If you have any questions or concerns regarding the program please feel free to contact me at my provided email.

\author{
Thank you, \\ Jennifer Fortes RN BSN \\ RIC NP Student \\ Jhoard_0674@email.ric.edu
}




\section{Appendix B}

\section{Informational Letter}

\section{Dear Fellow Nurses,}

For those of you who do not know me, my name is Jennifer Fortes and I am an RN at Charlton Memorial Hospital in the CVICU. I am also currently an Acute Care Nurse Practitioner Student at Rhode Island College.

Each student in this master's program is required to complete a master's level project regarding a topic they have selected. I have chosen to take a closer look at Noise in the Intensive Care Unit. In order to complete this quality improvement project, I am asking nursing staff in the Intensive Care (ICU) as well as Coronary Care (CCU) to participate.

This quality improvement project's purpose will be to evaluate nurses' knowledge and attitudes of the potentially harmful effects of noise on patients as well as themselves, and to identify opportunities for improvement with an aim of reducing noise in the intensive care unit. There will be a 10-15 minute in-service, which will take place on the unit during the beginning of January. This in-service will include an educational overview including hot points about noise in the ICU, definition of noise, the clinical impact in the hospital, and what we can do about reducing noise. There will be an anonymous test that will be provided to all participants during this in-service which will be completed before and after the in-service. It will take approximately 5 minutes to complete the pre-test, followed by the 10-15 minute in-service, and then 5 minutes to complete the post test. This will take no more than 25 minutes total of your time. Participation will be completely voluntary and anonymous. Informed consent will be 
implied when the participant reads this informational letter, completes the pre-test, and attends the educational inservice.

The findings of this project will be presented in a poster at the Rhode Island College School of Nursing Colloquium in May 2021. The results will also be available to all Critical Care staff via a poster on the unit. If you have any questions feel free to email me at jhoard_0674@email.ric.edu. Thank you in advance for your time and participation.

Jennifer Fortes, BSN, RN

Rhode Island College-ACNP Student 


\section{Appendix C}

Noise in the Intensive Care Unit Education

Content Outline:

Please complete the Noise in the Intensive Care Unit test labeled Pre-Test in front of you. Do not place any identifying or biographical data on the test. You will have five minutes to complete the test.

Learning Objectives

- Identify basic sources of noise in the hospital

- Identify adverse physical and psychosocial effects of noise on patients and staff members.

- Identify current levels of noise in the ICU

- Describe at least two strategies to reduce noise

Noise Hot Points!

- Noise in a busy acute care hospital, especially an Intensive Care Unit (ICU), is understandably present. It has been found that noises are often unpredictable and unexpected, as they have numerous sources.

- Florence Nightingale recognized noise as a health hazard dating as far back as 1859. She described noise as unnecessary as well as a cruel abuse of care.

- A variety of types of noises exist in the hospital, including things such as conversations, equipment alarms, telephones, pagers, televisions, closing doors, staff activities and so on. 
- Noise at high levels has been proven to have adverse effects on a patient's cardiovascular response, wound healing, gastric activity, pain management, sleep disturbances, and risk of ICU psychosis.

- Excessive noise can lead staff to experience anxiety, increased stress, annoyance, and increased rates of burnout. Ryherd et al. (2008) surveyed 47 nurses in a neurologic intensive care unit and found that $91 \%$ felt that noise negatively affected them in their daily work environment. Many reported experiencing noiseinduced stress symptoms such as irritation, fatigue, and headaches.

- Nurses spend far more time in the ICU than patients, meaning the nursing staff experience more exposure to excessive noise levels than patients, yet the longterm effects of noise on nursing staff is not well understood.

What is Noise?

- Noise has been described as an undesirable sound, lacking harmony and rhythm. Noise pollution has been defined as a level of environmental noise that is disturbing and obtrusive.

- Noise is commonly measured in a unit of measurement called the decibel (dB). Hospital Noise Clinical Impact

- According to the World Health Organization (WHO), noise levels should not exceed $35 \mathrm{dBs}$ (decibels at night and $40 \mathrm{~dB}$ during the day

- At $45 \mathrm{dBs}$, noise of normal living occurs including talking, or radio in the background. A noise level of $55 \mathrm{dBs}$ is equivalent to a noisy vacuum cleaner, a noisy lawn mower is equivalent to $60 \mathrm{dBs}$, and $85 \mathrm{dBs}$ is equivalent to a chainsaw. 
- An example of a sound source with a sound pressure level of $35 \mathrm{dBs}$ would be a very quiet room fan at low speed.

- Beginning at $40 \mathrm{dBs}$ learning or concentrating is possible, but distraction occurs.

- In a study by Darbyshire et al. (2013), sound levels in ICUs in the United Kingdom were consistently above WHO recommended limits and they discovered that noise levels above $60 \mathrm{dBs}$ occurred every minute.

What can we do?

- architectural-acoustic design

interventions such as sound absorbent material for ceilings, walls, and floors

- difficult to implement architecturally, and can be very costly.

- equipment design

- Purchase and use of quieter equipment, also costly.

- Staff Education

- A significant factor of inadequate noise control in the ICU is that the nurses have insufficient awareness regarding the hospital noise issue and its negative impact on even their own health status.

- Alarm reduction measures

- eliminating duplicate alarms, customizing alarms, changing electrocardiography electrodes daily, skin preparation, and using disposable electrocardiography leads

- Reduction in volume of pagers and telephones, adjust to vibrate when able. 
- Nurse-call systems. This is a team approach. Prevention of patients getting out of bed and setting off bed alarms requires assessment of patients needs prior to leaving the room

- 4 Ps: Potty, Position, Pain, Personal needs

○ Quiet Time Initiative

- Bundled care approach: Bundle your care with your patients in order to provide them a period of rest.

- Offer period of quiet time and "hands off"

- Offering Noise Canceling Headphones and Earplugs

- Music therapy to replace noxious sounds with pleasant ones: Care Channel at Southcoast

- Minimize conversations at centralized nursing station

Please complete the Noise in the Intensive Care Unit test labeled Post-Test in front of you. Do not place any identifying or biographical data on the test. You will have five minutes to complete the test. 


\section{Appendix D}

Noise In the Intensive Care Unit (ICU) Pre-Test

1. Noise can be defined as?
A. An undesirable sound
B. A sound lacking harmony and rhythm
C. Disturbing and obtrusive
D. All of the above

2. Noise is measured in?
A. Milliliters (ML)
B. Decibels $(\mathrm{dB})$
C. Ounces (OZ)
D. Kilograms $(\mathrm{Kg})$

3. The World Health Organization considers the acceptable range of noise level in the hospital to be?
A. should not exceed $25 \mathrm{~dB}$ at night and $30 \mathrm{~dB}$ during the day
B. should not exceed $45 \mathrm{~dB}$ at night and $50 \mathrm{~dB}$ during the day
C. should not exceed $35 \mathrm{~dB}$ at night and $40 \mathrm{~dB}$ during the day
D. should not exceed $55 \mathrm{~dB}$ at night and $60 \mathrm{~dB}$ during the day

4. Noise levels of normal living including talking, or radio in the background, occurs at what $\mathrm{dB}$ level?
A. $45 \mathrm{~dB}$
B. $30 \mathrm{~dB}$
C. $55 \mathrm{~dB}$
D. $75 \mathrm{~dB}$

5. At what noise level does distraction start to occur while learning and/or concentrating?
A. $30 \mathrm{~dB}$
B. $60 \mathrm{~dB}$
C. $40 \mathrm{~dB}$
D. $75 \mathrm{~dB}$

6. What is the average noise level near the nurse's station in the ICU?
A. $50-60 \mathrm{~dB}$
B. $30-40 \mathrm{~dB}$
C. $60-70 \mathrm{~dB}$
D. $70-80 \mathrm{~dB}$

7. Common acute physiologic change(s) that might be seen in a patient who is exposed to high noise levels is (are)?

A. Sleep Disturbance 

B. Elevated heart rate
C. Increased risk of ICU psychosis
D. Impaired pain management
E. All of the Above

8. All are effects of high noise level on ICU staff members EXCEPT?
A. Anxiety
B. Increased Stress
C. Decreased blood pressure
D. Increased rates of burnout

9. Which of the following is considered a staff behavior improvement strategy to reduce ICU noise levels?
A. Architectural acoustic design changes
B. Quieter equipment
C. Reduction in staff conversations at centralized nurses' station
D. Increased signage in the hospital to promote a quiet environment

10. Alarm reduction measures include all of the following, EXCEPT:
A. Individualize telemetry alarms
B. Answer call light whenever available, even if it is not your own
C. Change ECG electrodes daily
D. Assessing 4P's: Potty, position, pain, personal needs
E. None of the above 


\section{Appendix E}

\section{S Southcoast Health}

December 2, 2020

Jennifer Fortes, RN

363 Highland Ave

Fall River, Massachusetts 02720

Re: Assessing Nurses' Knowledge Toward Noise in The Intensive Care Unit: An Educational Intervention Project

Dear Ms. Fortes:

The Clinical Research Office of Southcoast Health received the request for the above referenced project that you would like to conduct within the Southcoast Health System. The Clinical Research Office (CTO) reviewed the information submitted with the Nursing Administration and has endorsed this clinical trial.

Southcoast does not have an internal IRB and therefore an external Central IRB listed on our Federal Wide Assurance (FWA) must be used. Southcoast Hospitals Group assurance number is FWA00009571.

You have chosen to use the Rhode Island College (RIC) Review Board as your governing IRB. Please submit a copy of this letter from the Southcoast Clinical Trials Office along with your study submission forms to RIC for their approval of the trial.

\section{Rhode Island College Review Board 600 Mount Pleasant Ave. \\ Providence, RI. 02903}

Please provide the Clinical Trials Office with a copy of the RIC approval/disapproval letter for the study when it is received. If you have any questions, please contact me at 508-973-7428 / isdaled@southcoast.org

Sincerely,

\section{debora Nedale}

Debora G. Isdale

Clinical Research Program Director

Southcoast Health 
Appendix F

Pre-Test Responses (Appropriate Response Bold)

\begin{tabular}{|c|c|c|c|c|c|c|c|c|c|c|c|}
\hline & Q1 & Q2 & Q3 & Q4 & Q5 & Q6 & Q7 & Q8 & Q9 & Q10 & Score \\
\hline 1 & D & B & A & B & $\mathbf{C}$ & D & $\mathbf{E}$ & $\mathbf{C}$ & $\mathbf{C}$ & $\mathrm{C}$ & $60 \%$ \\
\hline 2 & D & B & D & $\mathbf{A}$ & B & $\mathbf{A}$ & $\mathbf{E}$ & $\mathbf{C}$ & $\mathrm{C}$ & A & $70 \%$ \\
\hline 3 & D & B & A & B & $\mathbf{C}$ & $\mathbf{A}$ & $\mathbf{E}$ & $\mathbf{C}$ & $\mathbf{C}$ & $\mathrm{C}$ & $70 \%$ \\
\hline 4 & D & B & A & B & A & B & $\mathbf{E}$ & $\mathbf{C}$ & $\mathbf{C}$ & B & $50 \%$ \\
\hline 5 & D & B & B & $\mathbf{A}$ & B & $\mathrm{C}$ & $\mathbf{E}$ & $\mathbf{C}$ & $\mathbf{C}$ & B & $60 \%$ \\
\hline 6 & D & $\mathrm{C}$ & $\mathrm{C}$ & $\mathbf{A}$ & C & $\mathrm{C}$ & D & B & C & $\mathbf{E}$ & $50 \%$ \\
\hline 7 & A & B & A & $\mathrm{C}$ & $\mathbf{C}$ & $\mathrm{C}$ & $\mathbf{E}$ & A & $\mathbf{C}$ & $\mathbf{E}$ & $50 \%$ \\
\hline 8 & $\mathrm{C}$ & B & $\mathrm{D}$ & $\mathrm{C}$ & $\mathbf{C}$ & B & $\mathbf{E}$ & A & $\mathrm{C}$ & $\mathbf{E}$ & $50 \%$ \\
\hline 9 & D & B & A & $\mathrm{C}$ & A & $\mathrm{C}$ & B & $\mathrm{C}$ & $\mathrm{C}$ & $\mathbf{E}$ & $50 \%$ \\
\hline 10 & D & B & D & $\mathrm{C}$ & B & $\mathrm{C}$ & $\mathrm{C}$ & C & C & $\mathbf{E}$ & $50 \%$ \\
\hline 11 & $\mathrm{C}$ & B & $\mathrm{C}$ & $\mathbf{A}$ & B & $\mathrm{C}$ & $\mathrm{C}$ & B & $\mathrm{C}$ & $\mathbf{E}$ & $50 \%$ \\
\hline 12 & D & B & A & $\mathrm{C}$ & $\mathrm{A}$ & B & D & C & C & $\mathbf{E}$ & $50 \%$ \\
\hline 13 & D & B & $\mathrm{D}$ & $\mathrm{C}$ & A & B & $\mathbf{E}$ & $\mathrm{C}$ & $\mathrm{D}$ & $\mathbf{E}$ & $50 \%$ \\
\hline 14 & A & B & B & $\mathrm{C}$ & A & $\mathbf{A}$ & $\mathbf{E}$ & $\mathbf{C}$ & $\mathrm{D}$ & $\mathbf{E}$ & $50 \%$ \\
\hline 15 & D & B & B & $\mathrm{C}$ & B & $\mathbf{A}$ & $\mathbf{E}$ & $\mathrm{C}$ & $\mathrm{D}$ & $\mathbf{E}$ & $60 \%$ \\
\hline 16 & B & B & $A$ & B & $\mathbf{C}$ & B & $\mathbf{E}$ & $\mathbf{C}$ & $\mathrm{D}$ & $\mathbf{E}$ & $50 \%$ \\
\hline
\end{tabular}


Appendix G

Post-Test Responses (Appropriate Response Bold)

\begin{tabular}{|c|c|c|c|c|c|c|c|c|c|c|c|}
\hline & Q1 & Q2 & Q3 & Q4 & Q5 & Q6 & Q7 & Q8 & Q9 & $\mathrm{Q} 10$ & Score \\
\hline 1 & D & B & C & $\mathbf{A}$ & C & C & $\mathbf{E}$ & C & C & $\mathbf{E}$ & $90 \%$ \\
\hline 2 & D & B & C & A & B & A & $\mathbf{E}$ & C & C & $\mathbf{E}$ & $90 \%$ \\
\hline 3 & D & B & C & $\mathbf{A}$ & C & $\mathbf{A}$ & $\mathbf{E}$ & C & C & $\mathbf{E}$ & $100 \%$ \\
\hline 4 & D & B & A & $\mathbf{A}$ & C & $\mathrm{C}$ & $\mathbf{E}$ & C & C & $\mathbf{E}$ & $80 \%$ \\
\hline 5 & D & B & C & $\mathbf{A}$ & C & $\mathbf{A}$ & B & C & C & $\mathbf{E}$ & $90 \%$ \\
\hline 6 & D & B & C & $\mathbf{A}$ & C & $\mathbf{A}$ & $\mathbf{E}$ & C & C & $\mathbf{E}$ & $100 \%$ \\
\hline 7 & D & B & C & B & C & A & $\mathbf{E}$ & C & C & $\mathbf{E}$ & $90 \%$ \\
\hline 8 & D & B & C & $\mathbf{A}$ & B & $\mathbf{A}$ & $\mathbf{E}$ & C & C & $\mathbf{E}$ & $90 \%$ \\
\hline 9 & D & B & C & A & B & $\mathbf{A}$ & $\mathbf{E}$ & C & C & $\mathbf{E}$ & $90 \%$ \\
\hline 10 & D & B & A & $\mathbf{A}$ & C & A & $\mathbf{E}$ & C & C & $\mathbf{E}$ & $90 \%$ \\
\hline 11 & D & B & C & $\mathbf{A}$ & C & $\mathbf{A}$ & $\mathbf{E}$ & C & C & $\mathbf{E}$ & $100 \%$ \\
\hline 12 & D & B & $\mathbf{C}$ & $\mathbf{A}$ & C & $\mathbf{A}$ & $\mathbf{E}$ & C & $\mathbf{C}$ & $\mathbf{E}$ & $100 \%$ \\
\hline 13 & D & B & C & A & C & A & $\mathbf{E}$ & C & C & $\mathbf{E}$ & $100 \%$ \\
\hline 14 & D & B & C & A & C & A & $\mathbf{E}$ & C & C & $\mathbf{E}$ & $100 \%$ \\
\hline 15 & D & B & C & A & C & $\mathbf{A}$ & $\mathbf{E}$ & C & C & $\mathbf{E}$ & $100 \%$ \\
\hline 16 & D & B & C & A & C & A & $\mathbf{E}$ & C & C & $\mathbf{E}$ & $100 \%$ \\
\hline
\end{tabular}

